\title{
Energy-Efficient Smart Window Based on Thermochromic Hydrogel with Ultrahigh Visible Transparency and Unprecedented Infrared Transmittance Modulation
}

\section{Rong Zhang}

Nanjing Forestry University

\section{Bo Xiang}

Nanjing Forestry University

Min Feng

Jiangsu Academy of Agricultural Sciences

Liru Xia

Jiangsu Academy of Agricultural Sciences

Lei Xu

Jiangsu Academy of Agricultural Sciences

Shaochun Tang ( $\nabla$ tangsc@nju.edu.cn )

Nanjing University

Qingbao Guan

Donghua University https://orcid.org/0000-0002-3384-3229

\section{Article}

Keywords: Thermochromic Liquid Hydrogel, Ultrahigh Luminous Transmittance, Infrared Transmittance Modulation, Shape-independence

Posted Date: January 25th, 2021

DOl: https://doi.org/10.21203/rs.3.rs-134267/v1

License: (9) This work is licensed under a Creative Commons Attribution 4.0 International License. Read Full License 


\section{Abstract}

Both high visible transparency and strong solar modulating ability are highly required for energy-saving smart windows, but conventional responsive materials usually have low transparency and narrow solar transmittance range. Herein, we report a significant advance toward the design and fabrication of responsive smart windows by trapping novel V0.8W0.202@SiO2 doped poly(N-isopropyl acrylamide) (PNIPAm) thermochromic liquid hydrogel within two glass panels. The smart window is highly transparent to allow solar transmittance at low temperatures, while turns opaque automatically to cut off solar energy gain when exposed in sunlight. With a remarkably low content (1.0wt\%o) of dopant, V0.8W0.202@SiO2/PNIPAm (VSP) hydrogels exhibit ultrahigh luminous transmittance Tlum of $92.48 \%$ and solar modulation $\triangle$ Tsol of $77.20 \%$. The superior performance is mainly attributed to that V0.8W0.202@SiO2 doping induces PNIPAm particles' size reduction and internal structure change. Wdoping decreases the phase transition temperature (Tc) of VO2 from $68^{\circ} \mathrm{C}$ to $30^{\circ} \mathrm{C}$ (close to the Tc of PNIPAm), contributing to an unprecedented infrared transmittance modulation. Especially, the smart window shows excellent energy-saving during daytime outdoor demonstrations where practically achievable cooling temperature reaches up to $15.1^{\circ} \mathrm{C}$. In addition, the smart window exhibits outstanding stability, as embodied by unchanged optical performance even after 100 transparency-opaqueness reversible cycles. This new type of thermochromic hydrogel offering unique advantages of shapeindependence, scalability together with soundproof functionality promises potential applications in energy-saving buildings and greenhouses.

\section{Introduction}

Building energy consumption is as high as it even accounts for $40 \%$ of global energy consumption ${ }^{1,2}$. Serving as the main channel of heat exchange, windows are responsible for higher than $50 \%$ of the heat exchange energy between the buildings and the outside environment, and are thus the key to building energy saving ${ }^{3,4}$. The conventional energy-saving glasses need expensive equipment, thus developing high-performance and low-cost smart windows with unique advantages of shape independence, scalability, and soundproofing is highly desirable for energy-saving.

In recent years, growing demands for smart window that responds to temperature automatically to regulate the solar transmittance require advanced thermochromic materials (TCMs) as the most costeffective and energy-saving parts due to their rational stimulus and zero energy input properties. TCMs can selectively transmit, absorb or reflect specific spectra by means of changing its optical characteristics driven by external conditions (e.g., temperature, electric field, and illumination). Once they are used for smart window, besides control over color and transmittance, the indoor temperature can be effectively adjusted to realize energy saving. Ideally, an intelligent energy-saving smart window with both high visible transparency (solar transmittance) at low temperatures and strong solar modulating ability as well as thermal insulation at high temperatures is required. However, conventional responsive materials usually have low visible transparency and relatively narrow solar transmittance range, which cannot satisfy the practical requirements of smart windows. And most importantly, it is an inevitable 
challenge to further enhance the energy-saving capability of conventional TCMs due to their intrinsic performance limits ${ }^{5}$.

Among polymer TCMs candidates, poly(N-isopropyl acrylamide) (PNIPAm) stands out owing to its excellent optical switching performance ${ }^{6,7}$ and a low critical solution temperature (LCST) of around 32 ${ }^{\circ} \mathrm{C}^{8,9}$. Under external temperature stimuli, a rapid, discontinuous and reversible volume change of PNIPAm between the swollen and collapsed states leads to obvious transformations of its color and optical characteristics ${ }^{10}$. Unfortunately, the transmittance modulation of PNIPAm itself only covers a range of 350-1350 nm, much narrower than the entire solar spectral range of 300-2500 nm. Very recently, some strategies, such as graft copolymerization modification method ${ }^{11}$ and dispersing PNIPAm particles into heat-capacity water ${ }^{12}$ were adopted to improve the thermo-responsive optical properties of PNIPAm. Nevertheless, it is still a great challenge to obtain high visible transparency and strong solar modulating ability of PNIPAm simultaneously. For instance, the solar transmittance modulation ( $\left.\Delta T_{\text {sol }}\right)$ of PNIPAmbased smart windows could reach the maximal value of $81.3 \%$, while the luminous transmittance $\left(T_{\text {lum }}\right)$ value was much less than $90 \% 13,14$.

Integrating the PNIPAm with inorganic nanomaterials has been proved to be an effective route to extend the solar modulation range ${ }^{11}$. Vanadium dioxide $\left(\mathrm{VO}_{2}\right)$ is one of typical inorganic TCMs whose optical properties (especially the near-infrared transmittance) changes with $T_{\mathrm{c}}{ }^{12} \cdot \mathrm{VO}_{2}$ can adjust the transmittance of sunlight according to ambient temperature, and ultimately achieve a comfortable building environment of "warm winter and cool summer". Usually, the $\mathrm{VO}_{2}$-based devices show a high working temperature of $90^{\circ} \mathrm{C}$ or even $100^{\circ} \mathrm{C}$ due to the high metal-semiconductor $T_{\mathrm{C}}$ of $\mathrm{VO}_{2}\left(68^{\circ} \mathrm{C}\right)^{13,14}$, which leads to that most of reports focused on the spectral properties of $\mathrm{VO}_{2}-\mathrm{TCMs}$ only around $90^{\circ} \mathrm{C}$. In practical applications, however, the outdoor temperature of smart windows is much lower, especially the comfortable temperature for the human body is even less than $35^{\circ} \mathrm{C}$. So far, very little attention has been devoted to the temperature-involved studies, thus most of the reported TCMs are lacking of practicality and popularity. Sufficiently stable and efficient $\mathrm{VO}_{2}$-TCMs for practical use in smart windows have not been realized yet.

In order to address the issues, a variety of strategies including element doping ${ }^{15,16}$, nanocomposite construction $^{17,18}$, porosity controlling ${ }^{19,20}$, gridding ${ }^{21,22}$, and multilayer design were developed ${ }^{23,24}$. Among them, construction of composites at the nanoscale is one of the most efficient and conventional strategies to enhance $T_{\text {lum }}$ or/and $\Delta T_{\text {sol }}$. It was reported that combination of $\mathrm{VO}_{2}$ nanoparticles (NPs) with a thermo-responsive matrix led to that the $T_{\text {lum }}$ was increased up to $62.6 \%$, and the $\Delta T_{\text {sol }}$ value was also dramatically increased to $34.7 \%{ }^{1}{ }^{1,25}$ Problematically, heterogeneous interior structure made by classical synthesis results in high turbidity of the composite at room temperature. ${ }^{26}$ Additionally, reducing the $T_{\mathrm{c}}$ of $\mathrm{VO}_{2} \mathrm{NPs}$ in a $\mathrm{VO}_{2}$-TCMs composite material has been rarely investigated. Recently, a hydroxypropyl cellulose-based hydrogel was developed, which was endowed with excellent thermochromic performance ( $\Delta T_{\text {sol }}$ of $36.0 \%$ and $T_{\text {lum }}$ of $56.0 \%$ ) and relatively high $T_{\mathrm{c}}$ of $50{ }^{\circ} \mathrm{C}^{27}$. In 
spite of this, it is still urgent and challenging to develop a class of new TCMs with both high $T_{\text {lum }}$ and $\Delta T_{\text {sol, }}$ as well as a low $T_{\mathrm{c}}$ for efficient energy-saving ${ }^{28}$.

Herein, insight into trace doping of $\mathrm{V}_{0.8} \mathrm{~W}_{0.2} \mathrm{O}_{2} @ \mathrm{SiO}_{2}$ to maximize improvement of both high $T_{\text {lum }}$ and $\Delta T_{\text {sol }}$ of PNIPAm for energy-efficient smart window is proposed. Tungsten doping reduces the $T_{\mathrm{c}}$ of $\mathrm{VO}_{2}$ to $\sim 30{ }^{\circ} \mathrm{C}$, which is close to that of PNIPAm. The resulting $\mathrm{V}_{0.8} \mathrm{~W}_{0.2} \mathrm{O}_{2} @ \mathrm{SiO}_{2} /$ PNIPAm (VSP) have both ultrahigh visible transparency and unprecedented infrared transmittance modulation with extremely high $T_{\text {lum }}$ of $92.48 \%$ and $\Delta T_{\text {sol }}$ of $77.20 \%$. Particularly, the $\Delta T_{\text {sol }}$ value is higher than most reported values. The involved mechanisms of PNIPAm by $\mathrm{V}_{0.8} \mathrm{~W}_{0.2} \mathrm{O}_{2} @ \mathrm{SiO}_{2}$ doping and the strong synergy for the performance enhancement are discussed in detail. A novel type of responsive smart window is developed by trapping the liquid composite hydrogel within glass panels, and the smart window reached an achievable high outdoor temperature drop of $15.1^{\circ} \mathrm{C}$. The smart window with thermochromic VSP shows excellent stability with no obvious change of the spectral characteristics after 100 cycles. The thermoresponsive liquid trapped structure offers a disruptive strategy of easy fabrication, good uniformity, and scalability. The superior thermochromic capability of VSP together with soundproof functionality promises many applications in the field of highly energy-efficient smart windows, greenhouses and other thermochromic devices.

\section{Results}

Designed VSP hydrogel and TCM mechanism model. Fig. 1a schematically illustrates the switchable thermochromic behavior of the designed VSP hydrogel. At lower temperature (e.g., $\left.20^{\circ} \mathrm{C}\right)$, the window with VSP hydrogel is transparent to let in the solar transmittance; when heated from $20^{\circ} \mathrm{C}$ to $40^{\circ} \mathrm{C}$, the VSP hydrogel blocks sunlight automatically to cut off solar gain. Moreover, VSP maintains the excellent reversibility of the temperature-dependent spectra $\left(T_{\text {sol, } 20{ }^{\circ} \mathrm{C}}=78.82 \%, T_{\text {sol, } 40{ }^{\circ} \mathrm{C}}=1.62 \%\right)$. The newly developed VSP experienced a hydrophilic to hydrophobic transition at a low LCST. Below the LCST, the water molecules are kept in the interior of the PNIPAm macromolecules, which assures high transparency due to the high solar transmittance of the hydrogel. Once be heated above the LCST, the water molecules will be released from the PNIPAm, and the shrinkage particles will cause strong scattering of the light. The $\mathrm{V}_{0.8} \mathrm{~W}_{0.2} \mathrm{O}_{2} @ \mathrm{SiO}_{2}$ NPs in the VSP hydrogel plays an important role for enhancing the transmittance of sunlight and solar modulating ability. Particularly, the content of inorganic NPs used in this work was extremely lower than that in other similar reports ${ }^{1,22}$. The involved mechanism for the performance improvement will be discussed later.

Importantly, our as-prepared thermo-responsive VSP hydrogel is liquid in the form of gels and thus can be easily laminated in-between two layers of glasses to form a Glass-VSP-Glass type "sandwich" structure, which facilitates to regulate the transmittance of sunlight ${ }^{8,29}$ and its large-scale application for a smart window. Owing to the free-flowing feature, the liquid VSP has no any constraint of window shape. A thermo-responsive smart window using the liquid VSP was developed. Fig. 1b presents an illustration of the "sandwich" structure in which VSP hydrogel layer locates in-between two transparent glasses. When a 
building top is installed with suchlike large-area smart window for solar modulating, a high outdoor temperature drop will be realized and thus a comfortable building environment of "warm winter and cool summer" will be achievable. Interestingly, the trend of the transition state of the fabricated smart window with VSP shows strong time-dependence when illuminated under a $0.1 \mathrm{~W} / \mathrm{cm}^{2}$ infrared lamp. Fig. $1 \mathrm{c}$ shows the of the window at $505 \mathrm{~nm}$ after illumination for different times. Being irradiated for one minute, the window still maintained a high transmittance with the corresponding $T_{\text {lum }}$ value of $82.7 \%$. After four minutes, the window exhibited an opaque state with a very low value reaching $0.17 \%$. Obviously, the luminous transmittance $T_{\text {lum }}$ of the window gradually decreased with increasing the irradiation time. Fig. $1 \mathrm{~d}$ presents the corresponding photographs showing the time-dependent transmittance evolution of a VSP-based smart window device with an area of $10 \mathrm{~cm} \times 10 \mathrm{~cm}$. Within only $\sim 3$ minutes, it can be observed that the smart window changes from high transparency to opaqueness.

Construction of VSP. The synthesis process of VSP is illustrated in Fig. 2a. First, silica coated $\mathrm{V}_{0.8} \mathrm{~W}_{0.2} \mathrm{O}_{2}$ NPs were synthesized. Subsequently, the $\mathrm{V}_{0.8} \mathrm{~W}_{0.2} \mathrm{O}_{2} @ \mathrm{SiO}_{2} \mathrm{NPs}$ suspension in sodium dodecyl sulfate solution was added during the emulsion polymerization of PNIPAm, resulting in formation the VSP. Here the coating of silica enhances the stability of NPs and also the interaction between the groups on $\mathrm{SiO}_{2}$ coating and PNIPAm makes them to form a stable and uniform suspension. As shown in the scanning electron microscopy (SEM) images (Fig. S1a), the $\mathrm{V}_{0.8} \mathrm{~W}_{0.2} \mathrm{O}_{2}$ NPs have sizes of 20 50 nm, which is consistent with that obtained from a magnified transmittance electron microscopy (TEM) image (Fig. $\mathrm{S} 1 b)$. After $\mathrm{SiO}_{2}$ coating, the resulting $\mathrm{V}_{0.8} \mathrm{~W}_{0.2} \mathrm{O}_{2} @ \mathrm{SiO}_{2} \mathrm{NPs}$ with a core-shell structure exhibit clear outline and a uniform shell and their diameters increase to $\sim 70 \mathrm{~nm}$. The thickness of $\mathrm{SiO}_{2}$ shells is thus about $14 \mathrm{~nm}$ (Fig. S1d-e). The core-shell structure was further confirmed by energy dispersive spectrometer (EDS) mappings (Fig. S1c, S1f). The distributions of two main elements (V and W) in the VSP are also revealed by the EDS mapping (Fig. S4). Fig. 2b-d show TEM images at different maginfications of the resulting VSP, from which it can be seen that the NPs tend to be randomly distributed in the organic matrix. The $\mathrm{V}_{0.8} \mathrm{~W}_{0.2} \mathrm{O}_{2} @ \mathrm{SiO}_{2} \mathrm{NPs}$ are embedded in the 3D netwok of PNIPAm, which can be confirded by the EDS analysis.

XRD patterns of $\mathrm{VO}_{2}, \mathrm{~V}_{0.8} \mathrm{~W}_{0.2} \mathrm{O}_{2}$ and $\mathrm{V}_{0.8} \mathrm{~W}_{0.2} \mathrm{O}_{2} @ \mathrm{SiO}_{2}$ are shown in Fig. $2 \mathrm{f}$, where all the diffraction peaks are assigned to $\mathrm{VO}_{2}(\mathrm{M})$ with a monoclinic lattice symmetry and space group of $P 21 / C$ (JCPDS card No.43-1051). No other detectable impurity phases are observable in the pattern. To investigate the effects of tungsten ion $\left(\mathrm{W}^{6+}\right)$ doping and $\mathrm{SiO}_{2}$ on the $\mathrm{T}_{\mathrm{C}}$ of $\mathrm{VO}_{2}$, differential scanning calorimetry (DSC) analysis was performed. As shown in Fig. $2 \mathrm{~g}$, the $T_{\mathrm{c}}$ of $\mathrm{V}_{0.8} \mathrm{~W}_{0.2} \mathrm{O}_{2} \mathrm{NPs}\left(27^{\circ} \mathrm{C}\right)$ is much lower than that of $\mathrm{VO}_{2} \mathrm{NPs}\left(64.5^{\circ} \mathrm{C}\right)$, which is because the $\mathrm{W}^{6+}$ doping changes the position of $\mathrm{V}^{4+}$ and thus affects electronic states ${ }^{30}$. Meanwhile, the nano-effect makes that the $T_{\mathrm{c}}$ of $\mathrm{VO}_{2} \mathrm{NPs}$ is below the normal transition temperature of bulk $\mathrm{VO}_{2}\left(68^{\circ} \mathrm{C}\right){ }^{31,32}$. After the $\mathrm{SiO}_{2}$ coating, the $T_{\mathrm{c}}$ of $\mathrm{V}_{0.8} \mathrm{~W}_{0.2} \mathrm{O}_{2} @ \mathrm{SiO}_{2} \mathrm{NPs}$ $\left(27.7^{\circ} \mathrm{C}\right.$ ) is slightly higher (only $0.7^{\circ} \mathrm{C}$ increase) than that of $\mathrm{V}_{0.8} \mathrm{~W}_{0.2} \mathrm{O}_{2}$ (before coating), which is mainly 
attributed to the thermal insulation role of $\mathrm{SiO}_{2}$ layer ${ }^{14,33}$. The composition transition from $\mathrm{VO}_{2}$ to $\mathrm{V}_{0.8} \mathrm{~W}_{0.2} \mathrm{O}_{2}$ and to $\mathrm{V}_{0.8} \mathrm{~W}_{0.2} \mathrm{O}_{2} @ \mathrm{SiO}_{2}$ can be further verified by Fourier Transform Infrared Spectroscopy (FT-IR) analysis (Fig. S2). Also, the interactions between the groups on the $\mathrm{SiO}_{2}$ coated NPs and PNIPAm are demonstrated by the FT-IR analysis.

The thermochromic features are highly related to the particle size because the polymer affects the propagation path of light, leading to different visible light transmittance ${ }^{34}$. PNIPAm possesses both hydrophilic amide groups and hydrophobic isopropyl groups, which make it temperature-responsive. The main driving forces of phase transformation are hydrogen bonds and hydrophobicity. The hydrogen bonding force between the polymer chains and water molecules changes with temperature. The vector property of the hydrogen bond causes the polymer chains to stretch $(T<\mathrm{LCST})$ or shrink $(T>\mathrm{LCST})$ while leading to particle size change. The regularity of hydrogen bond is susceptible to temperature, and the hydrophilicity of the polymer chains are also changed, which results in a macroscopic change of VSP particle size distribution. Interestingly, the particle sizes of the VSP are much reduced comparing with the PNIPAm, while the LCST did not change. Addition of $\mathrm{V}_{0.8} \mathrm{~W}_{0.2} \mathrm{O}_{2} @ S i \mathrm{O}_{2}$ has a great influence on the polymerization process of PNIPAm, which results in an obvious decrease of particle size. However, the LCST is mainly related to the hydrogen bonding of PNIPAm. A decrease of the original density of PNIPAm network causes it fragmented into smaller particles under mechanical agitation. That is to say, the VSP induced size reduction of PNIPAm particles and microstructure change of 3D network of PNIPAm. The Wdoping decreases the $T_{\mathrm{c}}$ of $\mathrm{VO}_{2}$ from $68{ }^{\circ} \mathrm{C}$ to $\sim 30^{\circ} \mathrm{C}$ leading to an unprecedented infrared transmittance modulation range. The strong synergy between PNIPAm and NPs makes the smart window more sensitive to external temperature change.

Optical performance and theoretical calculations. Fig. 4a shows the transmittance spectra recorded at 20 ${ }^{\circ} \mathrm{C}$ and $40{ }^{\circ} \mathrm{C}$ of VSP containing different contents of $\mathrm{V}_{0.8} \mathrm{~W}_{0.2} \mathrm{O}_{2} @ \mathrm{SiO}_{2} \mathrm{NPs}$. At $20{ }^{\circ} \mathrm{C}$, as the $\mathrm{V}_{0.8} \mathrm{~W}_{0.2} \mathrm{O}_{2} @ \mathrm{SiO}_{2}$ content increases, the $\Delta T_{\text {sol }}$ of VSP shows a trend of gradual increase in the wavelength of $200 \sim 2500 \mathrm{~nm}$, especially in the visible region $(380 \sim 780 \mathrm{~nm})$. Based on the curves, the characteristic values (as listed in Table 1) are calculated by using the equations (1-2). The contents of $\mathrm{V}_{0.8} \mathrm{~W}_{0.2} \mathrm{O}_{2} @ \mathrm{SiO}_{2}$ in the VSP samples increases from zero to $1.0 \mathrm{wt} \%$ o. The $\Delta T_{\text {sol }}$ and $T_{\text {lum, } 20}{ }^{\circ} \mathrm{C}$ as the function of the solid contents are plotted in Fig. 4b. For the same VSP sample, the $\Delta T_{\text {sol }}$ at $40^{\circ} \mathrm{C}$ decreased significantly compared with that of $20^{\circ} \mathrm{C}$. When the content of $\mathrm{V}_{0.8} \mathrm{~W}_{0.2} \mathrm{O}_{2} @ \mathrm{SiO}_{2}$ are $0.4 \mathrm{wt} \%$, $0.6 \mathrm{wt} \%$ and $0.8 \mathrm{wt} \%$ o, the corresponding $T_{\text {lum }, 20}{ }^{\circ} \mathrm{C}$ values are $81.56 \%, 88.10 \%$ and $91.43 \%$, and the $\Delta T_{\text {sol }}$ are $73.10 \%, 77.20 \%$ and $61.11 \%$, respectively. Furthermore, increasing the solid content of $\mathrm{V}_{0.8} \mathrm{~W}_{0.2} \mathrm{O}_{2} @ \mathrm{SiO}_{2}$ from 0 to $1.0 \mathrm{wt} \%$ results in that the $T_{\text {lum, } 20}{ }^{\circ} \mathrm{C}$ increases proportionally. Notably, the $\Delta T_{\text {sol }}$ of the samples increases first and then declines, as shown in Fig. $4 \mathrm{~b}$. Once the solid content increases to higher than $0.6 \mathrm{wt} \%$, the $\Delta T_{\text {sol }}$ will be stable with no obvious change. It is worthwhile mentioning that the VSP exhibited drastically enhanced thermochromic properties as compared with that of the reported $\mathrm{VO}_{2}$-based thermochromic works (Table S1), as shown in Fig. 4c. In order to characterize 
the haze coefficient, the sample was also measured on the haze meter at $20^{\circ} \mathrm{C}$ and $40^{\circ} \mathrm{C}$, respectively. The results show that the haze of VSP IV was $2.53 \%\left(20^{\circ} \mathrm{C}\right)$ and $91.82 \%\left(40^{\circ} \mathrm{C}\right)$, consistent with the UVPC analysis results. This indicates that the optical properties of VSP are temperature sensitive.

\section{Discussion}

The uniqueness in light management of temperature-responsive PNIPAm particles is that their scattering behavior can be adjusted according to the internal structure, as illustrated in Fig. 4d. At low temperatures (below the LCST), the water molecules are within the PNIPAm macromolecules and lots of hydrogen bonds form between water and the PNIPAm molecular chains, which leads to a homogeneously low mismatch of refractive index between PNIPAm and water, and consequently a high solar transmittance. As the temperature increases, these hydrogen bonds are gradually broken, indicating a continuous phase separation. The oscillator strength of the $\mathrm{O}-\mathrm{H}$ bond is proportional to the hydrogen bond energy therefore, with fewer existing hydrogen bonds, the intensity of the transmittance valleys decreases. Once the temperature exceeds the LCST, the water molecules will be released from PNIPAm macromolecules and the polymer collapses, which results in a strong optical contrast to be established between PNIPAm and water, and the light is scattered at the interface between PNIPAm particls and surrounding water ${ }^{8,25}$, 35. Problematically, heterogeneous internal structure of PNIPAm particles made by the classical synthesis results in high turbidity of the hydrogel above LCST. To extend the transmittance modulation spectrum and guarantee the high $T_{\text {lum }}$, the hybrid strategy is to adjust the hydrogel particle size and the crosslinking density, as shown in Fig. 1a. After the introduction of $\mathrm{V}_{0.8} \mathrm{~W}_{0.2} \mathrm{O}_{2} @ \mathrm{SiO}_{2} \mathrm{NPs}$, the crosslinking density of PNIPAm reduces, which enhances the swelling capacity of the polymer. Therefore, much more hydrogen bonds form between water and the PNIPAm molecular chains and a higher solar transmittance is obtained at low temperature ${ }^{36,37}$. In general, When the hydrogel is below LCST, the transparency allows a large transmittance of solar radiation, whereas above LCST the scattering center induced by phase separation partially blocks radiation, thereby reducing transmittance.

Table 1. Thermochromic properties of VSP with different contents of $\mathrm{V}_{0.8} \mathrm{~W}_{0.2} \mathrm{O}_{2} @ \mathrm{SiO}_{2}$. 


\begin{tabular}{|lllllllll|}
\hline Sample & $\mathrm{V}_{\mathbf{0 . 8}} \mathrm{W}_{\mathbf{0 . 2}} \mathrm{O}_{2}$ & $T_{\text {lum }, 20{ }^{\circ} \mathrm{C}}$ & $T_{\text {lum }, 40}{ }^{\circ} \mathrm{C}$ & $\Delta T_{\text {lum }}$ & $T_{\text {sol, } 20{ }^{\circ} \mathrm{C}}$ & $T_{\text {sol,40 }}{ }^{\circ} \mathrm{C}$ & $\Delta T_{\text {IR }}$ & $\Delta T_{\text {sol }}$ \\
& $@ \mathrm{QSiO}_{2}(\mathrm{wt} \%)$ & $(\%)$ & $(\%)$ & $(\%)$ & $(\%)$ & $(\%)$ & $(\%)$ & $(\%)$ \\
\hline VSP I & 0 & 57.63 & 0.01 & 57.62 & 57.62 & 0.00 & 60.35 & 57.62 \\
\hline VSP II & 0.2 & 73.26 & 0.02 & 73.24 & 68.67 & 0.15 & 64.15 & 68.52 \\
\hline VSP III & 0.4 & 81.56 & 0.03 & 81.53 & 74.33 & 1.23 & 63.22 & 73.10 \\
\hline VSP IV & 0.6 & 88.10 & 0.04 & 88.06 & 78.82 & 1.62 & 63.47 & 77.20 \\
\hline VSP V & 0.8 & 91.43 & 11.21 & 80.22 & 81.19 & 20.08 & 35.17 & 61.11 \\
\hline VSP VI & 1.0 & 92.48 & 28.10 & 64.38 & 81.85 & 33.04 & 26.42 & 48.81 \\
\hline
\end{tabular}

In order to investigate the practical applications of our VSP hydrogel, a device with Glass-VSP-Glass type "sandwich" structure for smart window was assembled. The device was measured under $0.1 \mathrm{~W} / \mathrm{cm}^{2}$ continuous irradiation of an infrared lamp inside an infrared thermal insulation analyzer. A blank doublelayer glass was used for comparison. As shown in Fig. 5a, the initial temperatures of the VSP-based and blank double-layer glass are the same being $27.2^{\circ} \mathrm{C}$. Under the irradiation, the corresponding temperature of the devices started to rise gradually. It is obviously observed that the rate of temperature rising for our VSP-based device (on the right) is much lower than that of double-layer glass (on the left). After being illuminated for 500 seconds, their corresponding indoor temperatures of the VSP-based and blank doublelayer glass are $34.0^{\circ} \mathrm{C}$ and $49.1{ }^{\circ} \mathrm{C}$, respectively (Fig. 5b). Clearly, the VSP device can be cooled down by $15.1^{\circ} \mathrm{C}$ under $0.1 \mathrm{~W} / \mathrm{cm}^{2}$ irradiation. That their indoor temperatures change with the irradiation time is presented in the curve, as shown in Fig. 5c, and demonstrated by the Movie S1. After turning off the lamp, both the devices gradually returned to the initial temperature of $27.2^{\circ} \mathrm{C}$. Moreover, the thermal conductivity of our VSP hydrogels was evaluated. For example, the thermal conductivity of the VSP-IV hydrogel was measured to be $0.2678 \mathrm{~W} /(\mathrm{m} \cdot \mathrm{k})$, and its thermal diffusivity was measured to be 0.1262 $\mathrm{mm}^{2} / \mathrm{s}$. More importantly, the VSP device has the excellent reversibility of the temperature-dependent transmittance spectra at $550 \mathrm{~nm}$. That is to say, the change between the transparency with high light transmittance and opaqueness can be cycled between a temperature (e.g., $20^{\circ} \mathrm{C}$ ) and a high temperature (e.g., $40^{\circ} \mathrm{C}$ ), as shown in Fig. $5 \mathrm{~d}$. Even after 100 transparency-opaqueness reversible cycles, our VSP still exhibits the outstanding stability with no any change in optical performance.

The long-term anti-ultraviolet aging performance of our VSP hydrogel was measured. The transmittance spectra at $550 \mathrm{~nm}$ of VSP before and after UV irradiation was shown in Fig. S3. After UV irradiation for more than 100 hours, the transmittance of VSP does not decrease obviously, which indicates its excellent anti-ultraviolet aging ability. As demonstrated by the photographs, the window with VSP hydrogel is highly transparent to let in the solar transmittance at $20^{\circ} \mathrm{C}$ (as shown in Fig. $5 \mathrm{e}$ ), and turned to be opaque 
when the temperature increases to $40^{\circ} \mathrm{C}$ after irradiation of infrared light (Fig. 5f). In addtion, the VSP hydrogels have outstanding heat insulation properties, which promises their advantage of less thermal loss at high temperatures. Along with the excellent cooling performance, VSP is expected to play a key role in intelligent temperature control and would have hopeful prospects in energy-saving applications.

As shown in Fig. 6a, due to the unique advantage of liquid phase, VSP device can be completed with a simple pouring operation, which shows the easy fabrication as well as the high potential of scaling up and independence on window shape. Furthermore, the solution-phase synthesis described herein permits an industrial scale and cost-effective production, whereby a 30 ' $30-\mathrm{cm}$ window device is fabricated to display its scalability and the prospect of practical applications (Fig. 6b-c). For the large-scale sample, the VSP filled half of the double-glazed glass box $\left(30^{\prime} 15-\mathrm{cm}\right)$. Note that the photos of VSP device showing the change from high transparency to whloy opaqueness were recored outside of the buliding of College of Engineering and Applied Sciences of Nanjing University on September $8^{\text {th }}$ at Nanjing City, China $\left(32^{\circ} 7^{\prime} 16^{\prime \prime} \mathrm{N}, 118^{\circ} 57^{\prime} 19^{\prime \prime} \mathrm{E}\right)$. The simplicity of the single-component structure holds promise for industrial production and ease of implementation. The thermochromic hydrogel with the advantages of shapeindependence, scalability and soundproof functionality promises potential applications in energy-saving buildings.

In summary, a new type of intelligent temperature control device based on thermochromic VSP has been demonstrated. Especially by adjusting the content of $\mathrm{V}_{0.8} \mathrm{~W}_{0.2} \mathrm{O}_{2} @ S i \mathrm{O}_{2}$, the particle size and internal structure of VSP were effectively tailored, contributing to an ultra-high $\mathrm{T}_{\text {lum }, 20^{\circ} \mathrm{C}}$ of $92.48 \%$ (VSP-VI) and $\Delta \mathrm{T}_{\text {sol }}$ of $77.20 \%$. Meanwhile, doping of tungsten ions modifed the $T_{\mathrm{c}}$ of vanadium dioxide, which enabled it to exhibit a lower $T_{\mathrm{C}}$ of $30^{\circ} \mathrm{C}$. Thermochromic windows based on our VSP have reduced the indoor temperature by $15.1^{\circ} \mathrm{C}$, which also make VSP ideal for energy-saving. They maintain excellent stability after 100 cycles. The smart window may revolutionize the window industry based on their outstanding performance and good scalability for large-scale synthesis and commercial applications. Our findings provide an effective solution to regulate the transparency and thermal insulation of smart windows in response to environmental changes, which is of high potential to cut down the carbon emission and improve the sustainability of buildings and greenhouses. This revolutionary technology not only opens an avenue for revamping energy-saving smart windows but will also be broadly applicable to wearable sensors, optical modulators and medical devices.

\section{Methods}

Synthesis of $\mathrm{V}_{0.8} \mathrm{~W}_{0.2} \mathrm{O}_{2} \mathrm{NPs}$ : Chemicals used in the experiments are all analytical grade. Typically, vanadyl sulfate (Meryer Co., Ltd.) and ammonium paratungstate (Shanghai yuanye Bio-Technology Co., $L t d$.) were dissolved in distilled water (an atomic ratio of $V$ to $W$ was $4: 1$ ) to form a blue-green mixed solution. Then, $0.65 \mathrm{~mL}$ hydrazine monohydrate (50 vol\%, Ling-Feng Chemical Co., Ltd.) was slowly added dropwise by using a peristaltic pump. $\mathrm{pH}$ of the solution was adjusted to 8.0 by $0.1 \mathrm{M}$ of sodium hydroxide (Xilong Scientific Co., Ltd.), resulting in a brown precursor suspension. The precursor was 
centrifuged at $11,000 \mathrm{r} / \mathrm{min}$, and washed with distilled water for three times. Finally, it was transferred to a $100 \mathrm{~mL}$ Teflon-lined stainless-steel autoclave. Typically, the autoclave was maintained at $260^{\circ} \mathrm{C}$ for 36 hours. After cooling to room temperature (r. t.), the obtained $\mathrm{V}_{0.8} \mathrm{~W}_{0.2} \mathrm{O}_{2} \mathrm{NPs}$ were collected using a centrifuge and washed with distilled water and ethanol.

Synthesis of $V_{0.8} W_{0.2} O_{2} @ S i O_{2} N P s: 1.0 \mathrm{~g}$ of $\mathrm{V}_{0.8} \mathrm{~W}_{0.2} \mathrm{O}_{2}$ NPs were ultrasonically dispersed in $50 \mathrm{~mL}$ ethanol. Then, a solution composed of $280 \mathrm{~mL}$ ethanol and $70 \mathrm{~mL}$ aqueous ammonia (2 wt.\%, Sinopharm Chemical Reagent Co., $L t d$.) was added, and stirred for 30 minutes. Subsequently, $0.5 \mathrm{~mL}$ of tetraethyl orthosilicate was added through a peristaltic pump and stirred at $r$. t. for 4 hours. The powders were collected by centrifugation. $0.5 \mathrm{~g}$ of precursor powders were dispersed in $100 \mathrm{~mL}$ ethanol-ammonia aqueous solution (the volume ratio of ethanol to ammonia was 99:1). After that, $0.2 \mathrm{~mL} 3$ -

(trimethoxysilyl) propyl methacrylate (Aladdin Chemical Co., Ltd.) was added, and then stirred at r. t. for 4 hours. Finally, it was collected by centrifugation, and dried at $80^{\circ} \mathrm{C}$.

Synthesis of VSP Hydrogels: Typically, $5 \mathrm{mg}$ of dry $\mathrm{V}_{0.8} \mathrm{~W}_{0.2} \mathrm{O}_{2} @ \mathrm{SiO}_{2}$ powder was ultrasonically dispersed in $10 \mathrm{~mL}$ of $0.19 \mathrm{M}$ sodium dodecyl sulfate (Aladdin Chemical Co., Ltd.), and magnetically stirred at r. t. for 12 hours. Next, an aqueous solution containing $0.4 \mathrm{~g} \mathrm{~N}, \mathrm{~N}$-dimethylacetoacetamide (Aladdin Chemical Co., Ltd.) and $7 \mathrm{mg} \mathrm{N}, \mathrm{N}^{\prime}$-methylenebis (acrylamide) (Aladdin Chemical Co., Ltd.) was added into a fournecked flask with $200 \mathrm{~mL}$ of distilled water at $80{ }^{\circ} \mathrm{C}$ that was evacuated and purged with nitrogen. Under continuous stir, $1 \mathrm{~mL}$ sodium dodecyl sulfate solution containing $\mathrm{V}_{0.8} \mathrm{~W}_{0.2} \mathrm{O}_{2} @ \mathrm{SiO}_{2} \mathrm{NPs}$ was added into the flask. Subsequently, $0.8 \mathrm{~mL}$ ammonium persulphate aqueous solution was added to initiate the emulsion polymerization reaction, and the mixture was continuously stirred for 1 min. $3.5 \mathrm{~g} \mathrm{~N}, \mathrm{~N}$ dimethylacetoacetamide and $168 \mathrm{mg} \mathrm{N}$, N'-methylenebis (acrylamide) aqueous solution were dropwise added slowly for 2 hours. Finally, the resulting product of VSP hydrogels was obtained.

Materials Characterizations:The phase composition was characterized by X-ray diffractometer (XRD, D $\max / \mathrm{RB}, 12 \mathrm{~kW})$ with $\mathrm{Cu} K a$ radiation $(\lambda=0.15418 \mathrm{~nm})$, which were performed in the range of $20 \sim 80^{\circ}$ at a scanning rate of $10 \% \mathrm{~min}$. The time-dependent phase transition was measured via differential scanning calorimetry (DSC, DSC204 HP) in a continuous nitrogen flow from 10 to $90^{\circ} \mathrm{C}$ at a heating rate of 2 ${ }^{\circ} \mathrm{C} / \mathrm{min}$. The size distribution of nano-powders at variable temperatures was measured by dynamic light scattering analyzer (Zetasizer Nano ZS). Fourier Transform infrared spectroscopy (FT-IR) were recorded on an infrared spectrum meter (Nicolet iS50). The morphology and nanostructure were identified by using scanning electron microscopy (SEM, QUANTA250) and transmittance electron microscopy (TEM, Tecnai G20). The haze coefficient was conducted on the haze meter (Shenguang, WGT-S). Optical transmittance characteristics were measured with wavelengths ranging from 200 to $2600 \mathrm{~nm}$ at $20^{\circ} \mathrm{C}$ and $40{ }^{\circ} \mathrm{C}$ on a UV-Vis-NIR spectrophotometer (UVPC, Lambda 950). In detail, the thickness of the hydrogel sample was 2 $\mathrm{mm}$, and the thickness of the double glazing was also $2 \mathrm{~mm}$. The glass was ordinary silicate glass, the middle of the double glazing was air, and they were constructed using a silicone sealing adhesive. Based on the integral luminous transmittance $T_{\text {lum }}(380-780 \mathrm{~nm})$, IR transmittance $T_{\mathbb{I R}}(780-2500 \mathrm{~nm})$, and solar 
transmittance $T_{\text {sol }}(280-2500 \mathrm{~nm})$, the corresponding transmittance values were calculated from the equations (1) and (2):

$$
\begin{aligned}
& T_{\text {lum } / R / s o l}=\int \varphi_{\text {lum } / R / s o l}(\lambda) T(\lambda) \mathrm{d} \lambda / \int \varphi_{\text {lum } / R / s o l}(\lambda) \mathrm{d}(\lambda)
\end{aligned}
$$

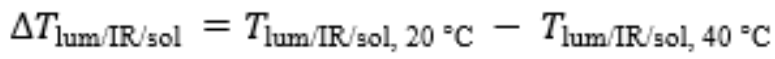

Where $T(\lambda)$ denotes the transmittance at wavelength $\lambda, \varphi_{\text {lum }}(\lambda)$ is the standard luminous efficiency function for the photopic vision, and $\varphi_{\text {sol }}(\lambda)$ is the solar irradiance spectrum for air mass 1.5 corresponding to the sun standing $37^{\circ}$ above the horizon.

\section{References}

1. Zhou Y, Cai Y, Hu X, Long Y. VO $\mathrm{V}_{2} /$ Hydrogel Hybrid Nanothermochromic Material with Ultra-High Solar Modulation and Luminous Transmission. J Mater Chem A 3, 1121-1126 (2015).

2. Ke Y, Zhou C, Yang Z, Wang S, Chan SH, Yi L. Emerging Thermal-Responsive Materials and Integrated Techniques Targeting the Energy-Efficient Smart Window Application. Adv Funct Mater 28, 1800113 (2018).

3. Gao Y, Luo H, Zhang Z, Kang L, Chen Z, Du J, Kanehira M, Cao C. Nanoceramic $\mathrm{VO}_{2}$ Thermochromic Smart Glass: a Review on Progress in Solution Processing. Nano Energy 1, 221-246 (2012).

4. Bell LE. Cooling, Heating, Generating Power, and Recovering Waste Heat with Thermoelectric Systems. Science 321, 1457-1461 (2008).

5. Ke Y, Chen J, Lin G, Wang S, Long Y. Smart Windows: Electro Thermo Mechano Photochromics, and Beyond. Advanced Energy Materials 9, 1902066 (2019).

6. Lee HY, Cai Y, Bi S, Liang YN, Song Y, Hu XM. A Dual-Responsive Nanocomposite toward ClimateAdaptable Solar Modulation for Energy-Saving Smart Windows. ACS App/ Mater Inter 9, 6054 (2017).

7. Yuan B, Wicks DA. Thermotropic color changing nanoparticles prepared by encapsulating blue polystyrene particles with a poly-N-isopropylacrylamide gel. Journal of Applied Polymer Science 105, 446-452 (2007).

8. Li X-H, Liu C, Feng S-P, Fang NX. Broadband Light Management with Thermochromic Hydrogel Microparticles for Smart Windows. Joule 3, 290-302 (2019).

9. Zhou Y, Cai Y, Hu X, Long Y. Temperature-responsive hydrogel with ultra-large solar modulation and high luminous transmission for "smart window" applications. Journal of Materials Chemistry A 2, 13550-13555 (2014).

10. Ma H, Zhu M, Luo W, Li W, Fang K, Mou F, Guan J. Free-standing, flexible thermochromic films based on one-dimensional magnetic photonic crystals. J Mater Chem C 3, 2848-2855 (2015).

11. Wang Y, Zhao F, Wang J, Khan AR, Guo X. VO ${ }_{2} @ S_{2}{ }_{2} /$ Poly(N-isopropylacrylamide) Hybrid Nanothermochromic Microgels for Smart Window. Ind Eng Chem Res 57, (2018). 
12. Fang $\mathrm{X}$, Xun $\mathrm{C}$, Luo H, Ping J. Recent Advances in $\mathrm{VO}_{2}$-Based Thermochromic Composites for Smart Windows. J Mater Chem C 6, 1903-1919 (2018).

13. Kang L, Gao Y, Luo H, Chen Z, Du J, Zhang Z. Nanoporous Thermochromic $\mathrm{VO}_{2}$ Films with Low Optical Constants, Enhanced Luminous Transmittance and Thermochromic Properties. ACS App/ Mater Inter 2, 135-138 (2011).

14. Zhu J, Zhou Y, Wang B, Zheng J, Ji S, Yao H, Luo H, Jin P. Vanadium Dioxide Nanoparticle-Based Thermochromic Smart Coating: High Luminous Transmittance, Excellent Solar Regulation Efficiency, and Near Room Temperature Phase Transition. ACS Appl Mater Inter 7, 27796-27803 (2015).

15. Nan S, Shi C, Zhang C, Liu X, Cao C, Dong B, Luo H, Liu J, Gao Y. The Synthesis and Performance of Zr-Doped and W-Zr-Co doped $\mathrm{VO}_{2}$ Nanoparticles and Derived Flexible Foils. J Mater Chem A 2, 15087-15093 (2014).

16. Wang N, Duchamp M, Dunin-Borkowski RE, Liu S, Zeng X, Cao X, Long Y. Terbium-Doped $\mathrm{VO}_{2}$ Thin Films: Reduced Phase Transition Temperature and Largely Enhanced Luminous Transmittance. Langmuir 32, 759-764 (2016).

17. Gao Y, Wang S, Luo H, Dai L, Cao C, Liu Y, Zhang C, Kanehira M. Enhanced Chemical Stability of $\mathrm{VO}_{2}$ Nanoparticles by the Formation of $\mathrm{SiO}_{2} / \mathrm{VO}_{2}$ Core/Shell Structures and the Application to Transparent and Flexible $\mathrm{VO}_{2}$-Based Composite Foils with Excellent Thermochromic Properties for Solar Heat Control. Energ Environ Sci 5, 6104-6110 (2012).

18. Kim H, Yang S. Responsive Smart Windows from Nanoparticle-Polymer Composites. Adv Funct Mater 30, 1902597 (2020).

19. Xun C, Ning W, Yan LJ, Loo SCJ, Shlomo M, Yi L. Nanoporous Thermochromic $\mathrm{VO}_{2}(\mathrm{M})$ Thin Films: Controlled Porosity, Largely Enhanced Luminous Transmittance and Solar Modulating Ability. Langmuir 30, 1710-1715 (2014).

20. Ning W, Huang Y, Magdassi S, Mandler D, Hai L, Yi L. Formation of $\mathrm{VO}_{2}$ ZeroDimensional/Nanoporous Layers with Large Supercooling Effects and Enhanced Thermochromic Properties. Rsc Adv 3, 7124-7128 (2013).

21. Ke Y, Wen X, Zhao D, Che R, Xiong Q, Long Y. Controllable Fabrication of Two-Dimensional Patterned $\mathrm{VO}_{2}$ Nanoparticle, Nanodome, and Nanonet Arrays with Tunable Temperature-Dependent Localized Surface Plasmon Resonance. Acs Nano 11, $7542-7551$ (2017).

22. Shen N, Chen S, Wang W, Shi R, Chen P, Kong D, Liang Y, Amini A, Wang J, Cheng C. Joule Heating Driven Infrared Switching in Flexible $\mathrm{VO}_{2}$ Nanoparticle Films with Reduced Energy Consumption for Smart Windows. J Mater Chem A 7, 4516-4524 (2019).

23. Zheng J, Bao S, Ping J. $\mathrm{TiO}_{2}(\mathrm{R}) / \mathrm{VO}_{2}(\mathrm{M}) / \mathrm{TiO}_{2}(\mathrm{~A})$ Multilayer Film as Smart Window: Combination of Energy-Saving, Antifogging and Self-Cleaning Functions. Nano Energy 11, 136-145 (2015).

24. $\mathrm{Koo} \mathrm{H}$, Shin D, Bae SH, Ko KE, Chang SH, Chan P. The Effect of $\mathrm{CeO}_{2}$ Antireflection Layer on the Optical Properties of Thermochromic $\mathrm{VO}_{2}$ Film for Smart Window System. J Mater Eng Perform 23, 402-407 (2014). 
25. Zhou Y, Cai Y, Hu X, Long Y. Temperature-Responsive Hydrogel with Ultra-Large Solar Modulation and High Luminous Transmission for "Smart Window" Applications. J Mater Chem A 2, 13550-13555 (2014).

26. Zhu J, Huang A, Ma H, Chen Y, Zhang S, Ji S, Bao S, Ping J. Hybrid Films of $\mathrm{VO}_{2}$ Nanoparticles and A Nickel(II)-Based Ligand Exchange Thermochromic System: Excellent Optical Performance with a Temperature Responsive Colour Change. New J Chem 41, 830-835 (2017).

27. Yang YS, Zhou Y, Chiang FBY, Long Y. Tungsten Doped $\mathrm{VO}_{2} /$ Microgels Hybrid Thermochromic Material and its Smart Window Application. Rsc Adv 7, 7758-7762 (2017).

28. Cui Y, Ke Y, Liu C, Chen Z, Wang N, Zhang L, Zhou Y, Wang S, Gao Y, Long Y. Thermochromic $\mathrm{VO}_{2}$ for Energy-Efficient Smart Windows. Joule 2, 1707-1746 (2018).

29. Zhou Y, Layani M, Wang S, Hu P, Ke Y, Magdassi S, Long Y. Fully Printed Flexible Smart Hybrid Hydrogels. Adv Funct Mater 28, (2018).

30. Sun C, Yan L, Yue B, Liu H, Gao Y. The Modulation of Metal-Insulator Transition Temperature of Vanadium Dioxide: a Density Functional Theory Study. J Mater Chem C 2, 9283-9293 (2014).

31. Dai L, Cao CX, Gao YF, Luo HJ. Synthesis and Phase Transition Behavior of Undoped $\mathrm{VO}_{2}$ with a Strong Nano-Size Effect. Sol Energ Mat Sol C 95, 712-715 (2011).

32. Li Y, Qi W, Yuan L, Janssens E, Huang B. Modeling the Size-Dependent Solid-Solid Phase Transition Temperature of $\mathrm{Cu}_{2} \mathrm{~S}$ Nanosolids. J Phys Chem C 116, 9800-9804 (2012).

33. Li S, Li Y, Jiang M, Ji S, Luo H, Gao Y, Jin P. Preparation and Characterization of Self-Supporting Thermochromic Films Composed of $\mathrm{VO}_{2}(\mathrm{M}) @ S_{2} \mathrm{O}_{2}$ Nanofibers. ACS Appl Mater Inter 5, 6453-6457 (2013).

34. Li X, Liu C, Feng S, Fang N. Broadband Light Management with Thermochromic Hydrogel Microparticles for Smart Windows. Joule 3, 1-13 (2019).

35. Schild HG. Poly( $\mathrm{N}$-isopropylacrylamide): experiment, theory and application. Progress in Polymer ence 17, 163-249 (1992).

36. Zhou Y, Cai Y, Hu X, Long Y. VO ${ }_{2}$ /hydrogel hybrid nanothermochromic material with ultra-high solar modulation and luminous transmission. J Mater Chem A 3, 1121-1126 (2014).

37. Contreras-Cáceres R, Schellkopf L, Fernández-López C, Pastoriza-Santos I, Pérez-Juste J, Stamm M. Effect of the Cross-Linking Density on the Thermoresponsive Behavior of Hollow PNIPAM Microgels. Langmuir 31, 1142-1149 (2014).

\section{Declarations}

\section{Acknowledgements}

The authors acknowledge financial support from National Natural Science Foundation of China (32001429, 51703148), China Postdoctoral Science Foundation (2019M661768, 2020M671442), the 
Natural Science Foundation of Jiangsu Province (Grant No. BK20161396), the Key Research and Development Program of Jiangsu Provincial Department of Science and Technology of China (BE2020684), Jiangsu Planned Projects for Postdoctoral Research Funds (2019K091, 2020Z369), Scientific Research Start-up Funds of Nanjing Forestry University (163101127), and the Fundamental Research Funds for the Central Universities $(14380163,14913411)$.

\section{Author contributions}

R.Z. performed the analysis and drafted the manuscript. S.T. conceived the idea, designed the research. B.X. carried out theoretical calculations and designed the figures. L.X. and M.F. contributed to the interpretation of the data. L.X. and Q.G. revised the manuscript.

\section{Competing interests}

The authors declare no competing interests.

\section{Figures}


(a)
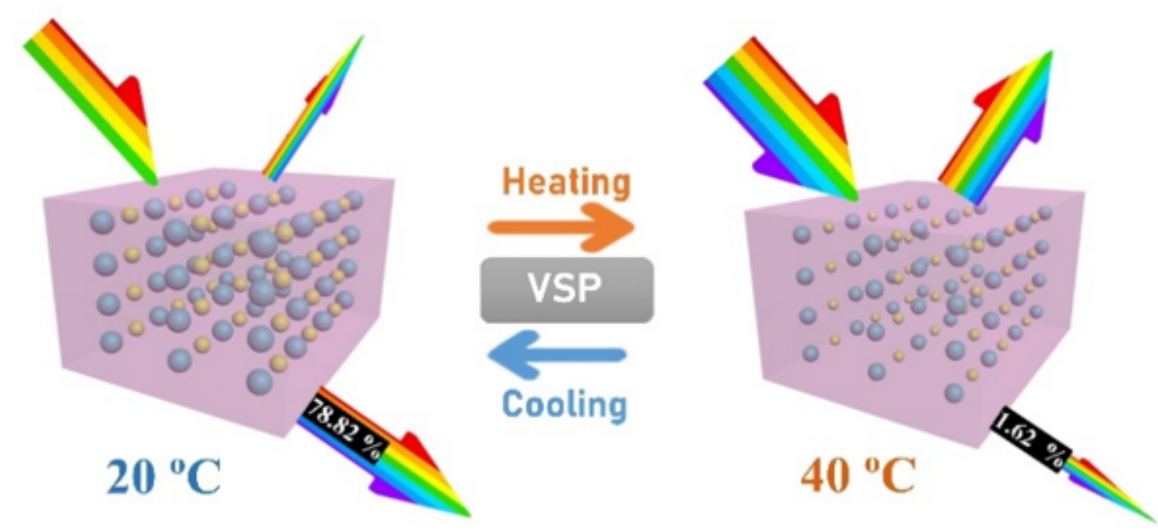

(b)
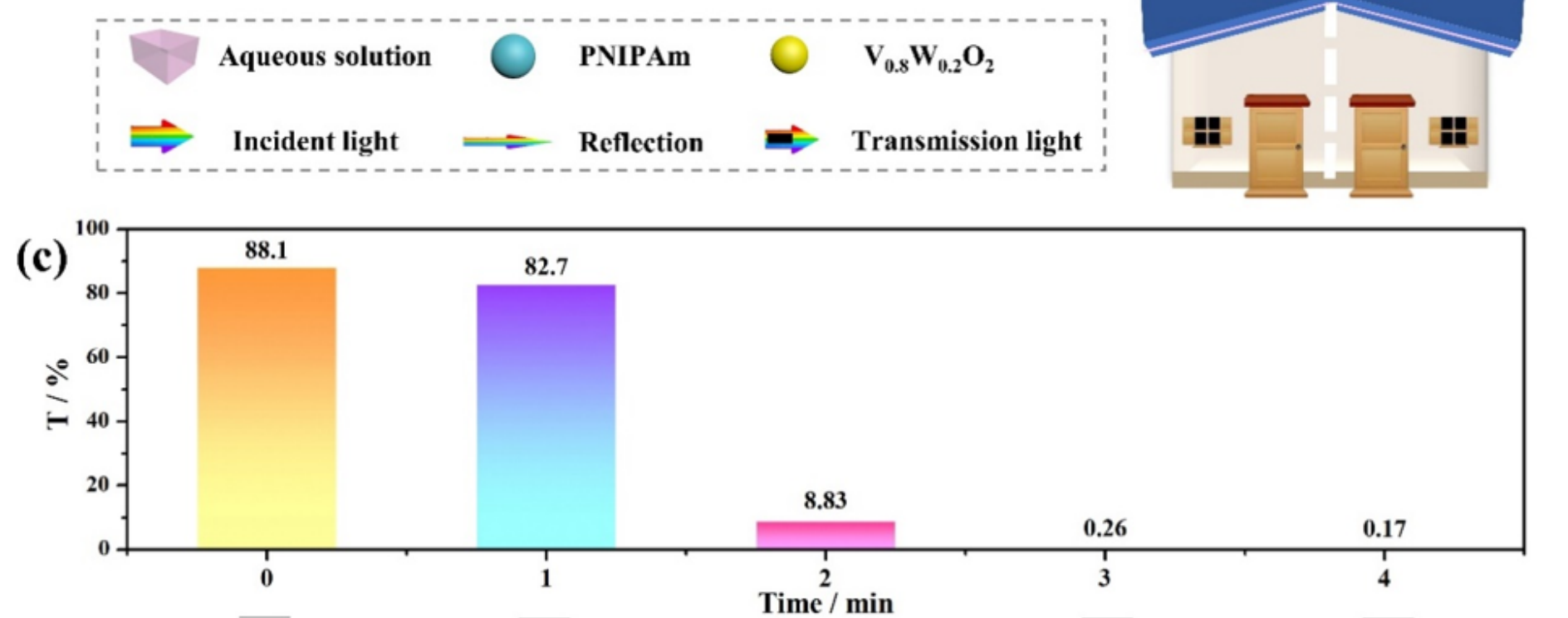

(d)
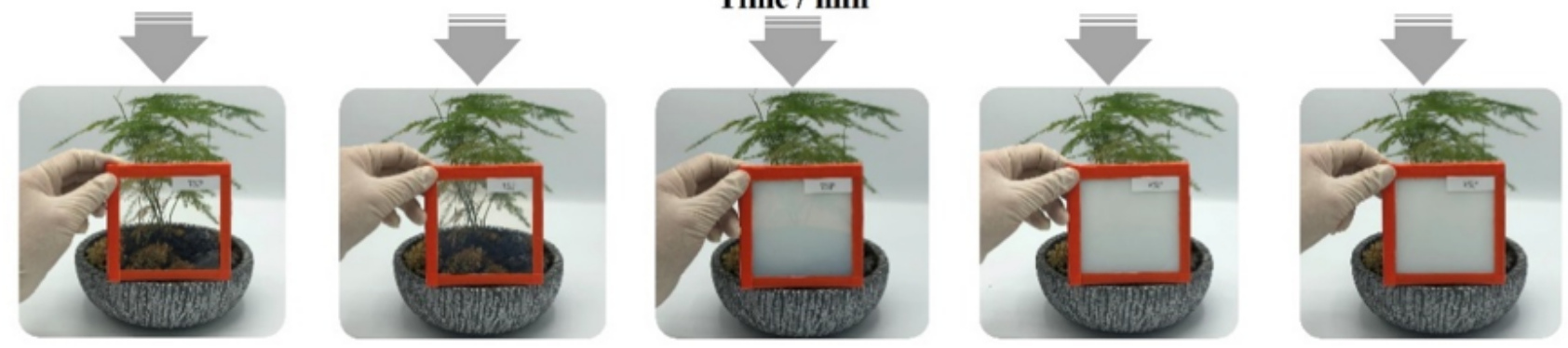

\section{Figure 1}

(a) Schematic illustration of the switchable thermochromic behavior of the designed VSP hydrogel. (b) illustration of the "sandwich" structure in which VSP hydrogel layer locates in-between two transparent glasses. Thermochromic application of the smart window on a building top leading to a comfortable building environment of "warm winter and cool summer". (c) Statistical analysis of the transmittance (at $505 \mathrm{~nm}$ ) at different irradiation times, and (d) the corresponding photographs showing the timedependent transmittance evolution of a smart window device with an area of $10 \mathrm{~cm} \times 10 \mathrm{~cm}$. 
(a)
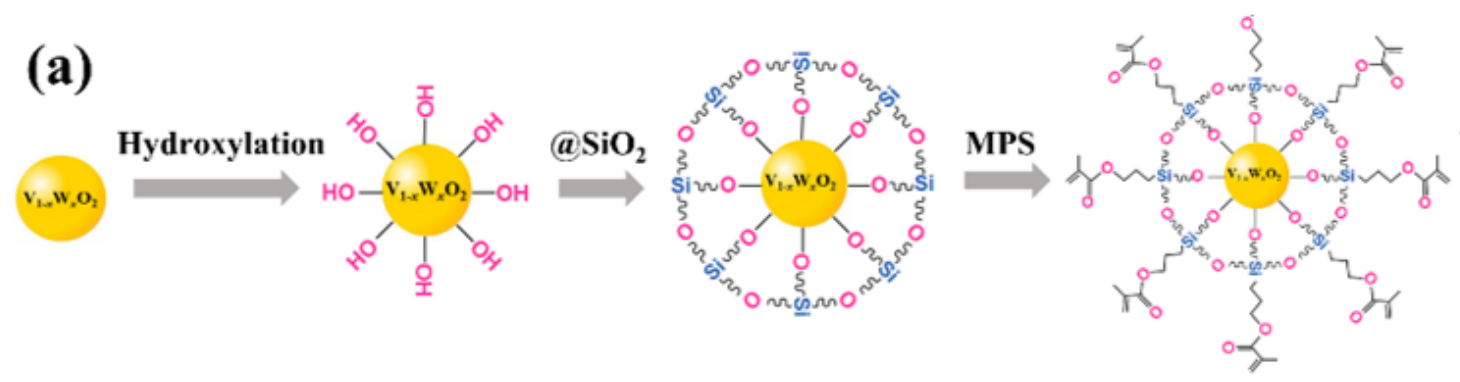

PNIPAm
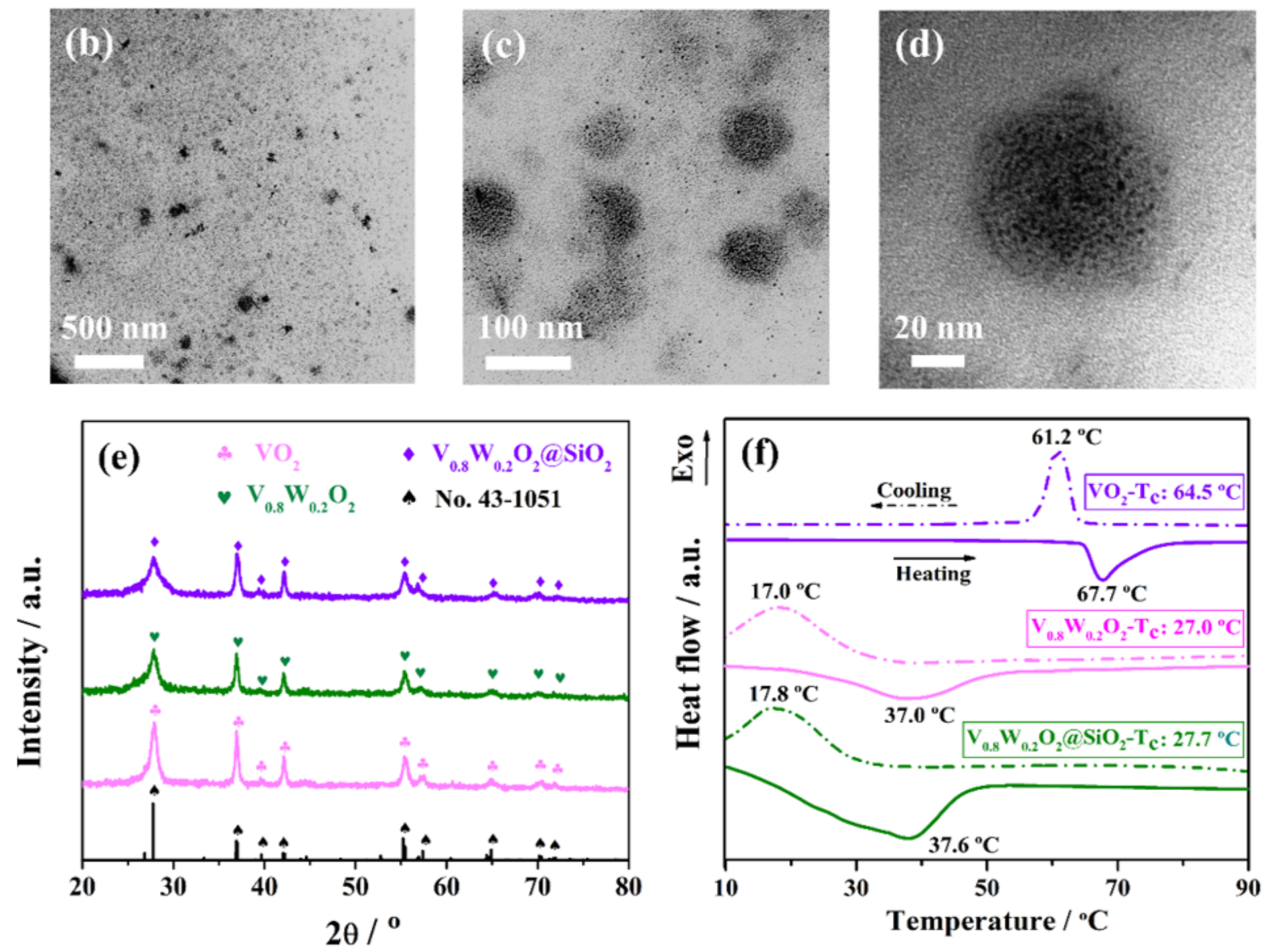

Figure 2

A schematic diagram of the preparation process. (a) Schematic illustration of the fabrication procedure of organic-inorganic nanocomposites, (b)-(d) TEM images at different magnifiactions of VSP, and (e) the element distribution of VSP obtained by Energy Dispersive Spectrometer (EDS). (f) XRD patterns of pure VO2, V0.8W0.2O2 and V0.8W0.202@SiO2 NPs, and (g) their DSC thermograms. 

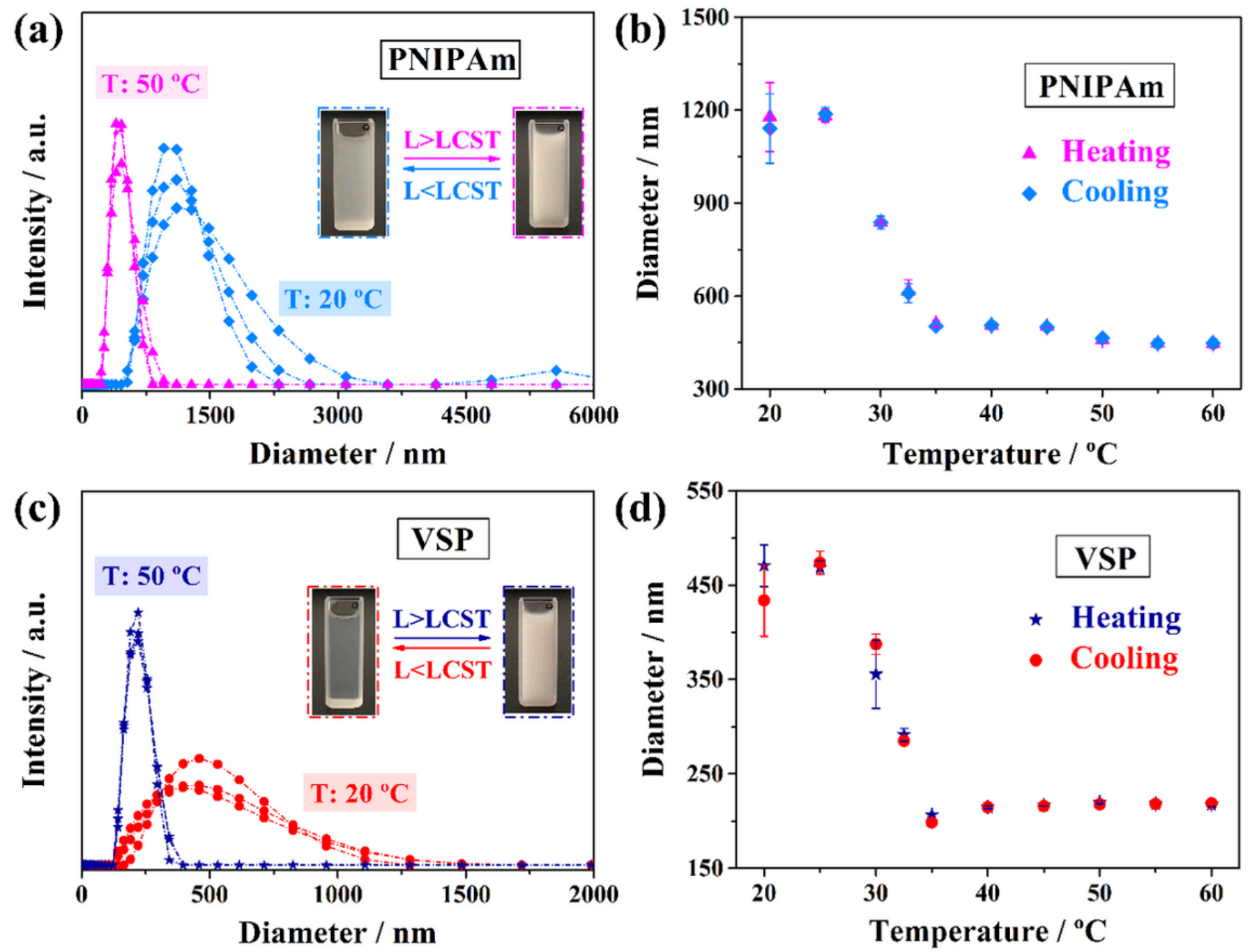

Figure 3

Temperature-Responsive Properties of the PNIPAm and VSP hydrogels. $(a, b)$ Hydrodynamic diameters of PNIPAm and (c,d) VSP at different temperatures. Insets of (a) and (c) are the photographs showing transparency change of the PNIPAm and VSP hydrogels. 
(a)

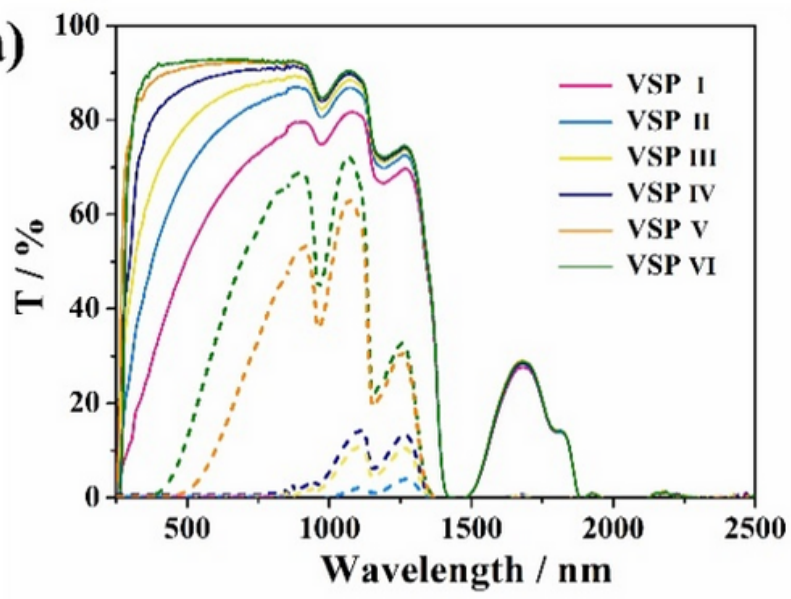

(c)

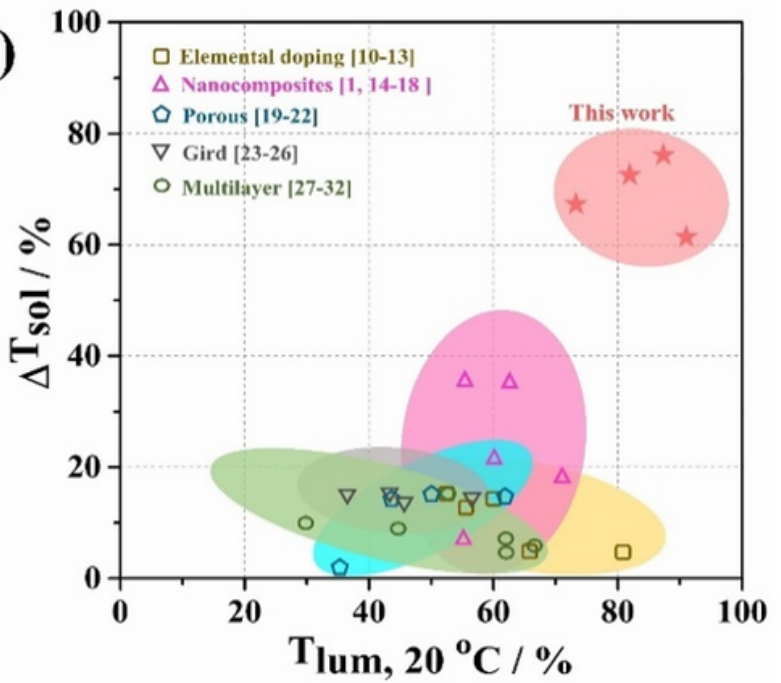

(b)

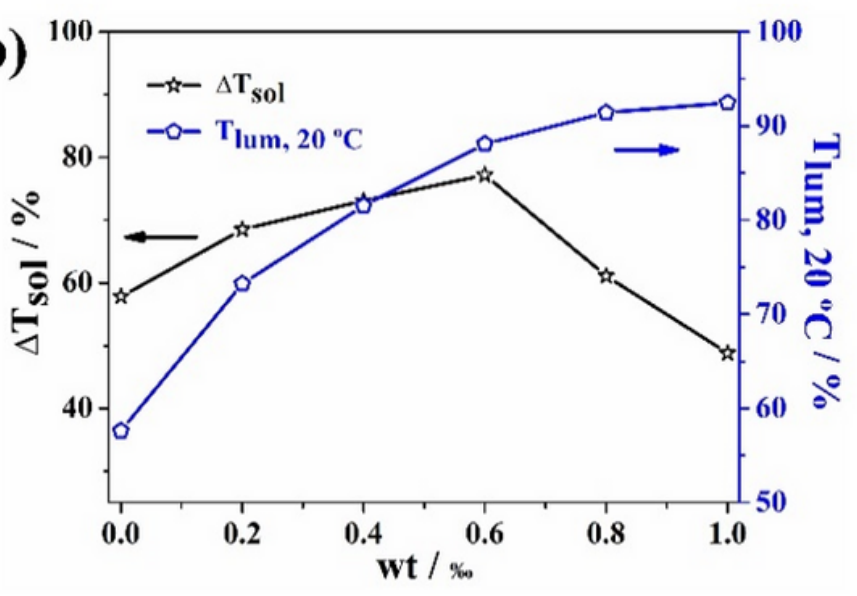

(d)
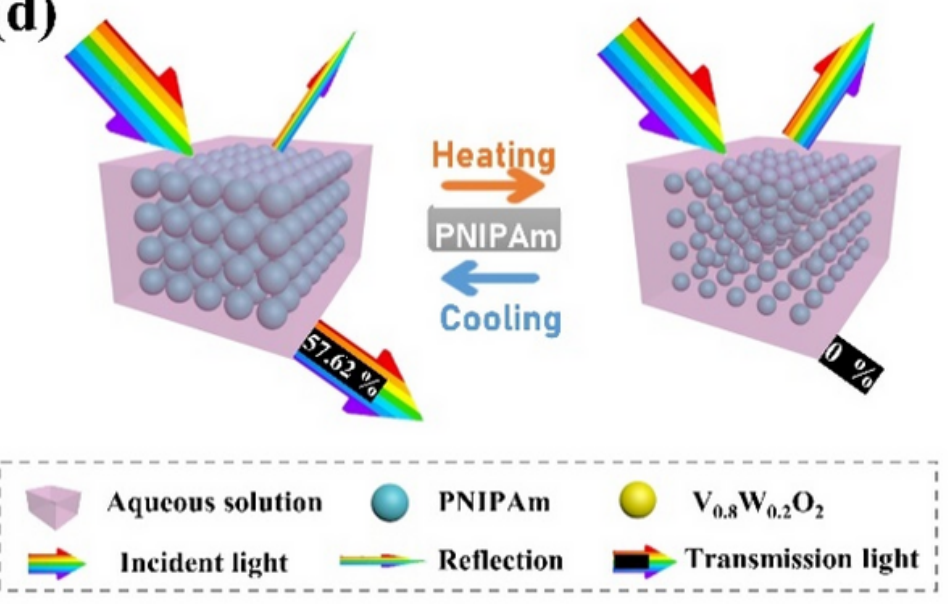

\section{Figure 4}

Thermochromic property of PNIPAm with different contents of V0.8W0.202@SiO2. (a) The transmittance spectra of PNIPAm with different solid contents of V0.8W0.202@SiO2 (in different colors) at $20^{\circ} \mathrm{C}$ (solid lines) and $40^{\circ} \mathrm{C}$ (short dash lines). (b) $\triangle \mathrm{Tsol}$ and Tlum, $20^{\circ} \mathrm{C}$ as a function of solid contents of V0.8W0.202, wherein the symbols were calculated by the equations (1) and (2) according to the curves of Fig. 4a. (c) Summary of representative samples with the best thermochromic performance ( $\Delta$ Tsol and Tlum, $20^{\circ} \mathrm{C}$ ) in the literature. (d) Thermochromic model and schematic diagram of PNIPAm, from left to right: PNIPAm- $20^{\circ} \mathrm{C}$, PNIPAm- $40{ }^{\circ} \mathrm{C}$. 
(a)

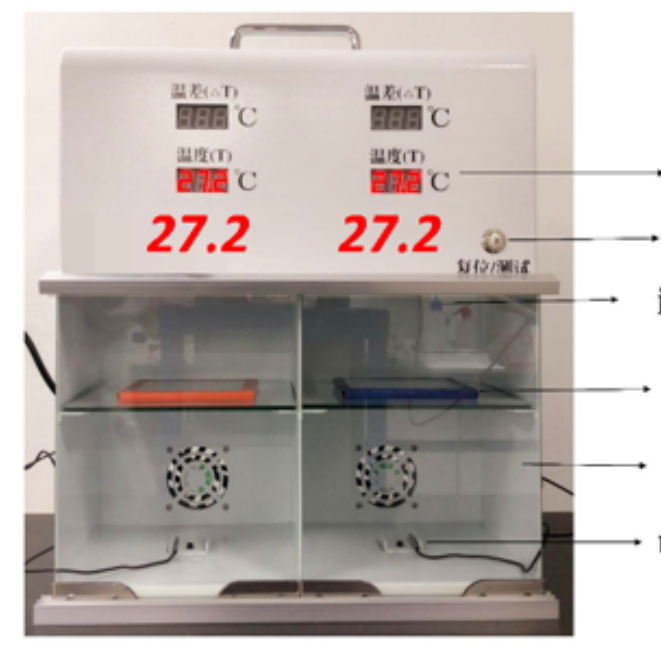

(c)

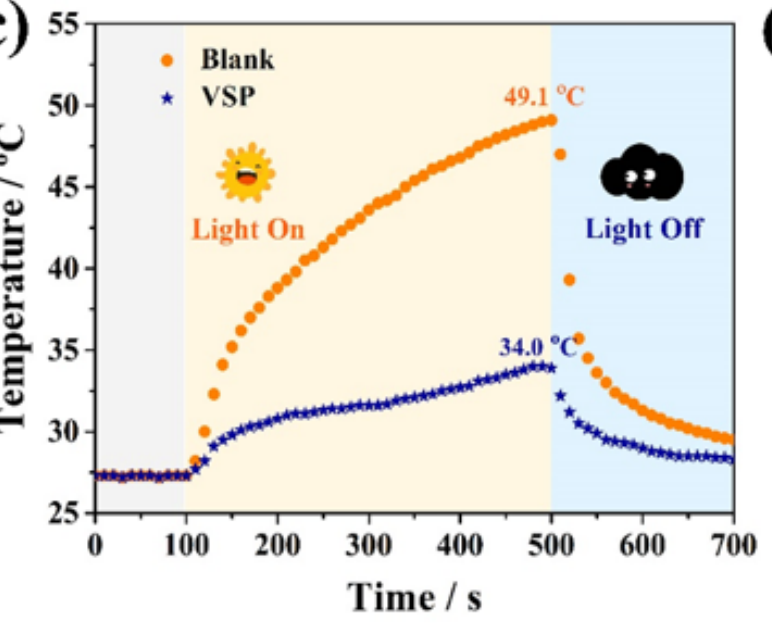

(e)

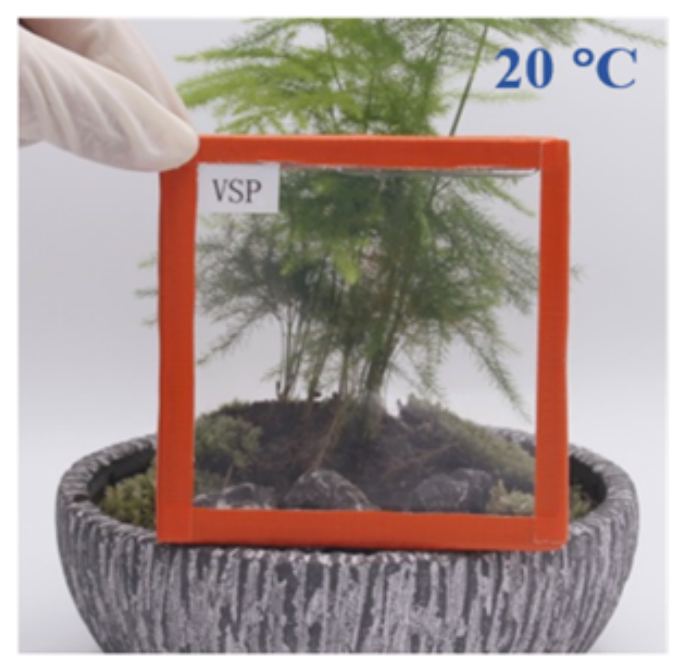

(b)

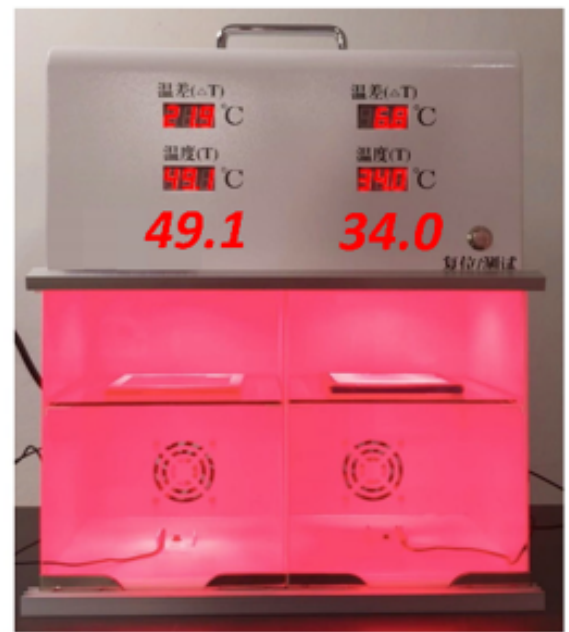
detector

(d)

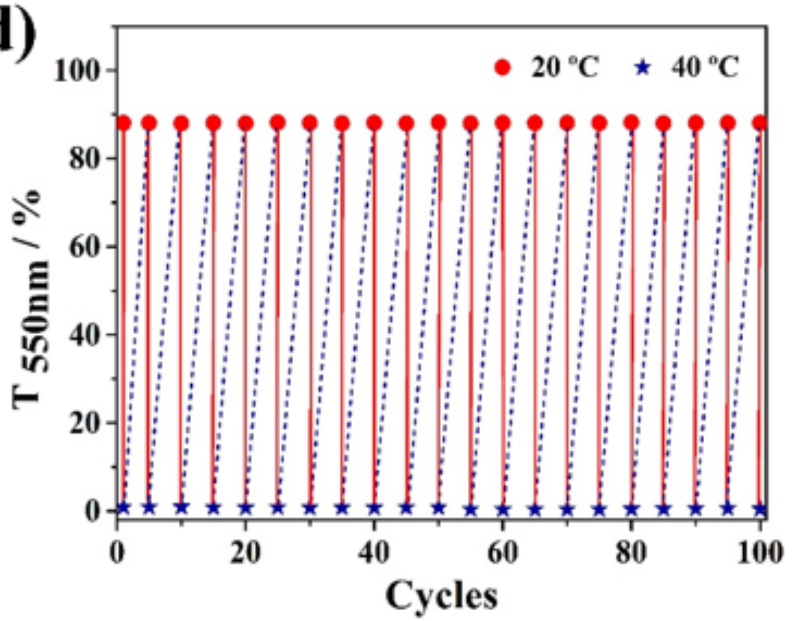

(f)

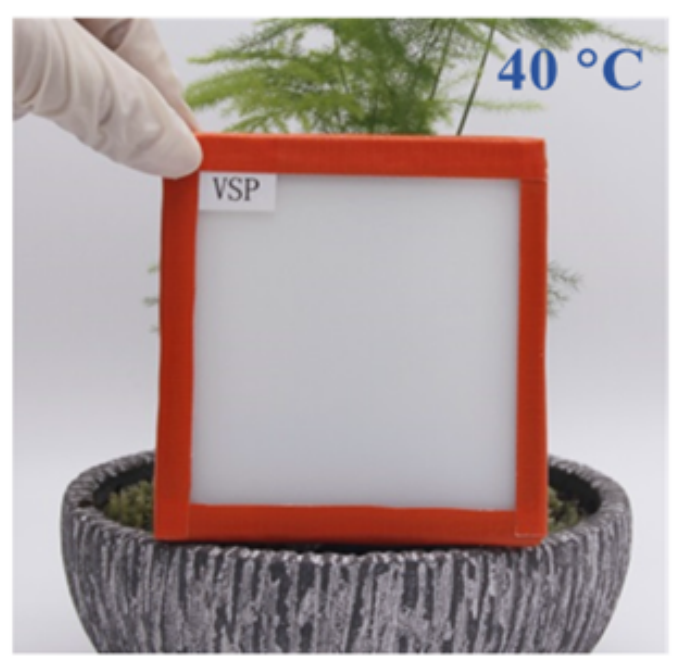

\section{Figure 5}

The cooling performance of VSP and its application as a new thermochromic material in smart window.

(a) The experimental setup for measurements of the VSP at the intial and final temperatures. As the sandwich of double-layer glass, VSP was compared with blank double-layer glass under the irradiation of infrared lamp $(0.1 \mathrm{~W} / \mathrm{cm} 2)$, and their corresponding indoor temperature as a function of time. (b) The results of the VSP, glass composite device and a blank double-layer glass placed in the infrared thermal 
insulation analyzer (left: VSP and glass composite device, right: blank double-layer glass). (c) the measured indoor temperatures change curve with the irradiation time. (d) Visible light transmittance distribution at $550 \mathrm{~nm}$ for VSP during 100 continuous cycles. Photographs of glass sandwich structure with VSP at (e) $20^{\circ} \mathrm{C}$ and (f) $40^{\circ} \mathrm{C}$ (after irradiation of infrared light).
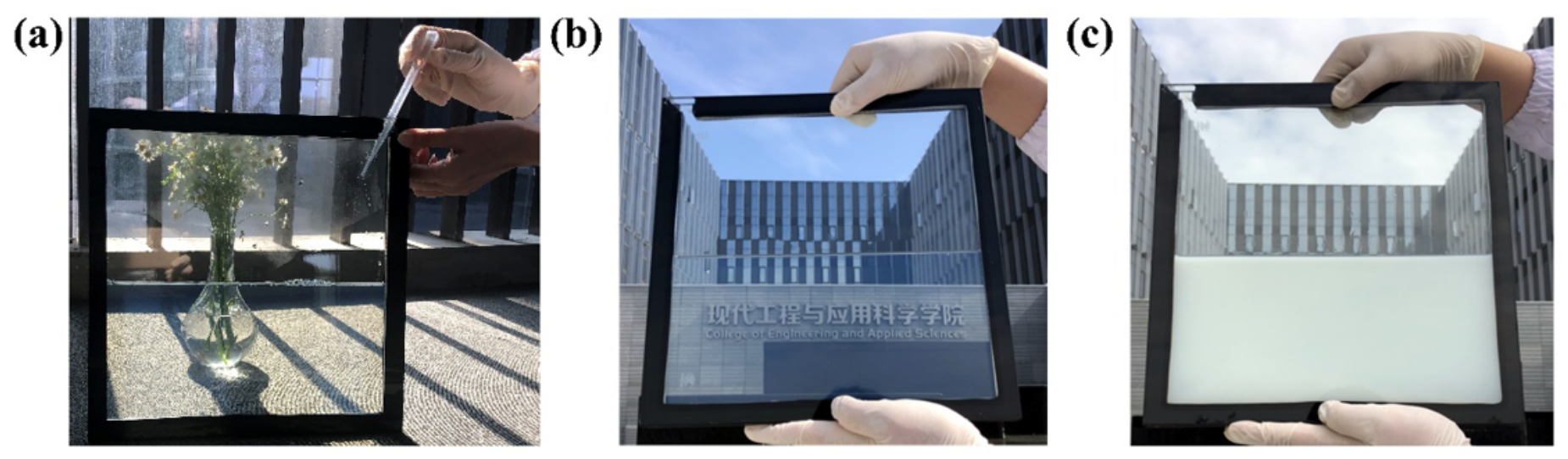

Figure 6

Photographs showing large-sized VSP Device of $30 \mathrm{~cm}$ X $30 \mathrm{~cm}$ and its change under sunlight. (a) The process of filling the hollow layer of laminated glass with VSP liquid. The photos of VSP device were recored outside of the buliding of College of Engineering and Applied Sciences of Nanjing University on September 8th at Nanjing City, P. R. China ( $\left.32^{\circ} 7^{\prime} 16^{\prime \prime} N, 118^{\circ} 57^{\prime} 19^{\prime \prime} E\right)$. (b) Before phase transition. (c) After phase transition under outdoor sunlight.

\section{Supplementary Files}

This is a list of supplementary files associated with this preprint. Click to download.

- MovieS1.mp4

- Supportinglnformation.doc 